\title{
Adverse Childhood Experiences and Criminal Propensity among Intimate Partner Violence Offenders
}

\author{
N. Zoe Hilton, Elke Ham and Michelle M. Green
}

Version Post-print/accepted manuscript

Citation Zoe Hilton, N., Ham, Elke \& M. Green, Michelle. (2016). Adverse

(published version) Childhood Experiences and Criminal Propensity Among Intimate Partner Violence Offenders. Journal of Interpersonal Violence. $10.1177 / 0886260516674943$.

How to cite TSpace items

Always cite the published version, so the author(s) will receive recognition through services that track citation counts, e.g. Scopus. If you need to cite the page number of the author manuscript from TSpace because you cannot access the published version, then cite the TSpace version in addition to the published version using the permanent URI (handle) found on the record page.

This article was made openly accessible by $U$ of $T$ Faculty. Please tell us how this access benefits you. Your story matters. 
Journal of Interpersonal Violence, Advanced online publication September 2016

\author{
Adverse Childhood Experiences and Criminal Propensity among \\ Intimate Partner Violence Offenders \\ N. Zoe Hilton, Elke Ham and Michelle M. Green \\ Waypoint Centre for Mental Health Care
}

Author Note

N. Zoe Hilton, Ph.D., Department of Psychiatry, University of Toronto, Toronto, Ontario, Canada, and Waypoint Research Institute, Waypoint Centre for Mental Health Care, Penetanguishene, Ontario, Canada; Elke Ham, Waypoint Research Institute, Waypoint Centre for Mental Health Care, Penetanguishene, Ontario, Canada; Michelle Green, Ph.D., Forensic Assessment Program, Waypoint Centre for Mental Health Care, Penetanguishene, Ontario, Canada.

We would like to thank Carol Lang, Jenna Rutherford, and Sonja Dey for research assistance. We also thank Terri Newman, Louise Moreau, and Waypoint Provincial Forensic Programs staff for administrative assistance and helpful feedback on this research, and Waypoint Clinical Information Services staff for assistance. Angela Eke, Alecia Dretzkat, and the late Marnie Rice provided helpful comments on drafts of this manuscript.

Correspondence concerning this article should be addressed to Dr. N. Zoe Hilton, Senior Research Scientist, Waypoint Centre for Mental Health Care, 500 Church Street, Penetanguishene, ON, L9M 1G3, Canada. Email: zhilton@waypointcentre.ca, zoe.hilton@utoronto.ca 


\begin{abstract}
Background: Adverse childhood experiences (ACEs), defined as exposure to abuse and adverse household events, are prevalent among certain offenders including those who commit intimate partner violence (IPV). However, it is not clear how ACEs relate to criminal propensity among IPV offenders, who have been shown to exhibit less antisociality and institutional violence than other offenders. Method: We compared 99 male offenders with a current or previous offense of IPV with 233 non-IPV violent offenders and 103 nonviolent offenders undergoing institutional forensic assessment. This convenience sample allowed for use of extensive psychosocial records as well as study of institutional violence. Results: IPV offenders had the highest mean ACE score and more extensive criminal propensity on some measures (violent and nonviolent criminal history and psychopathy) than both other groups. ACEs were associated with most measures of criminal propensity in the whole sample but with only one (actuarial risk of violent recidivism) in the subsample of IPV offenders. Conclusion: Finding that ACEs are prevalent among IPV offenders even in this sample with extensive mental illness demonstrates the robustness of this phenomenon. IPV offenders, though, are similar to other violent offenders in this respect and there is insufficient evidence that ACEs represent a criminogenic need among IPV offenders specifically. Further research could draw from the batterer typology literature and attend to IPV offenders' broader criminal careers.
\end{abstract}


Adverse Childhood Experiences and Criminal Propensity among Intimate Partner Violence Offenders

Adverse Childhood Experiences (ACEs) are defined as verbal, physical, or sexual abuse and exposure to adverse events in the household including substance abuse, mental illness, intimate partner violence, and criminal behavior (Felitti et al., 1998). ACEs are associated with risk of serious physical, mental health, and neurobiological problems, and more exposure predicts greater risk (e.g., Bellis, Lowey, Leckenby, Hughes, \& Harrison, 2013; Björkenstam et al., 2013; Felitti et al., 1998; Fergusson, Boden \& Horwood, 2008a; Kelly-Irving et al., 2013; Mersky, Topitzes, \& Reynolds, 2013; Teicher \& Samson, 2016). Exposure is often measured by an ACE score that sums the number of events (e.g., Finkelhor, Shattuck, Turner, \& Hamby, 2013). Most people report at least one adverse childhood experience (e.g., Dong et al., 2004) but ACEs appear elevated among offenders (e.g., Reavis, Looman, Franco, \& Rojas, 2013), especially men who commit sexual offenses (e.g., Jesperson, Lalumière, \& Seto, 2009; Levenson \& Socia, 2015) or intimate partner violence (IPV) (e.g., Crane, Oberleitner, \& Easton, 2013; Cunha \& Gonçalves, 2013; Henrichs, Bogaerts, Sijtsema, \& Klerx-van Mierlo, 2015; Mair, Cunradi, \& Todd, 2012; Milaniak \&Widom, 2015; Watt \& Scrandis, 2013; Whitfield, Anda, Dube, \& Felitti, 2003). Exposure to IPV in childhood is associated with a higher rate of other ACEs (e.g., Dube, Anda, Felitti, Edwards, \& Williamson, 2002).

Relevant research among IPV offenders has been limited to community samples and it is not yet evident whether ACEs are elevated among IPV offenders in correctional or forensic psychiatric institutions. Research with such offenders can inform correctional practice, particularly if ACEs are related to criminal propensity and merit prioritization for intervention. In the present study, we examined ACEs among men with a current or previous offense of IPV 
undergoing forensic assessment in an institutional unit. We compared them with similar offenders without an IPV history on the ACEs documented in their detailed clinical record. Our study aimed to extend the current literature on ACEs among IPV offenders, which has mostly focused on the childhood abuse features of ACEs, and drew from research on IPV offenders' criminal propensity in order to investigate whether ACEs would be associated with criminal propensity in a clinical sample of offenders and among those charged with IPV in particular.

\section{ACEs and IPV Offenders}

Since the first attention to ACEs among adult IPV perpetrators (e.g., Whitfield et al., 2003), evidence has accumulated for an association between IPV and abuse, witnessing domestic violence, and parental criminal offending (e.g., Capaldi, Knoble, Shortt, \& Kim, 2012;

Fergusson, Boden, \& Horwood, 2008b; Mair et al., 2012). Longitudinal studies have shown that ACEs self-reported at age 17 or 21 predicted IPV among men and women by age 25 (Fergusson et al., 2008b) and that childhood abuse or neglect predicts IPV perpetration (Milaniak \& Widom, 2015) even after controlling for other adverse experiences (e.g., Millett, Kohl, Jonson-Reid,

Drake, \& Petra, 2013). It has been suggested that ACEs may be an important treatment target for some offenders (e.g., Levenson \& Socia, 2015). The link between childhood abuse and IPV has long been attributed to social learning processes (e.g., Smith-Marek et al., 2015) which could inform treatment for this type of adverse event. However, studies to date on IPV offenders are limited and some questions that could inform a treatment approach remain to be addressed.

Much research into ACEs among IPV offenders is limited to physical, sexual, or other forms of abuse in childhood, whereas the complete picture of ACEs includes parental crime, substance abuse, mental illness, and other adverse household events. Research into the full range of ACEs could better inform appropriate offender intervention. For example, treatment for abuse 
histories might focus on the cumulative effects of trauma (e.g., Henrichs et al., 2015; Levenson \& Socia, 2015), whereas evidence that parental substance abuse may play a role in offenders' antisociality might suggest a benefit of integrated treatment for IPV and substance abuse (e.g., Corvo \& Carpenter, 2000; Radatz \&Wright, 2015). There is, therefore, a need for improved understanding of ACEs among IPV offenders. The present study of offenders undergoing institutional assessment examined not only documented childhood abuse but also other adverse household events including parental mental illness, substance abuse, and criminality.

\section{Criminal Propensity among IPV Offenders}

Criminal propensity can be conceptualized in various ways, such as criminal history, measures of risk, or psychopathy. Psychopathy encompasses interpersonal and affective characteristics (e.g., callousness, shallow affect) as well as antisocial behaviors (e.g., conning and manipulating others, criminal versatility) and as such it has been conceptualized as indicating criminal propensity or proclivity and hypothesized as an underlying mechanism of criminal behavior (e.g., Krupp, Sewall, Lalumière, Sheriff, \& Harris, 2013). Psychopathy has been consistently associated with violent and nonviolent criminal behavior (e.g., Harris, Rice, Quinsey, \& Cormier, 2015; Salekin, Rogers, \& Sewell, 1996).

Analyzing data from a long-term cohort study including both self-report and criminal records, Theobald, Farrington, Coid, and Piquero (2015) reported that men who committed only IPV $(n=46)$ had lower scores on every facet of psychopathy than men who had a violent conviction only $(n=50)$. Offenders who committed both IPV and had convictions for other violence $(n=21)$ scored higher still. Theobald and colleagues' sample was drawn from a community cohort. Hilton et al. (2001) reported similar results in a forensic institutional sample using criminal records and psychosocial assessment reports. Among the 508 men, those whose 
index offenses included IPV $(n=88)$ scored lower on the Psychopathy Checklist-Revised (PCLR; Hare, 2003). They also scored lower on every item of the Violence Risk Appraisal Guide (VRAG; Harris et al., 2015), an actuarial assessment of risk for violent recidivism which includes PCL-R score and 11 other items. The reason for lower psychopathy and recidivism risk among IPV offenders is not clear, but research into different facets of psychopathy among offenders in a county jail indicates that IPV offenders may exhibit more affective deficits but less impulsivity than others (Swogger, Walsh, \& Kosson, 2007).

IPV offenders also exhibit lower criminal propensity than other violent offenders when measured in terms of institutional violence. Sorensen, Vigen, Woods, and Williams (2015) compared 189 IPV homicide and 111 home-invasion homicide offenders. IPV offenders were less likely to assault other inmates or staff, or to commit other serious rule violations (e.g., weapons, cell phones, drugs, threats of harm, escape attempts, rioting). Institutional aggression provides a useful additional measure of criminal propensity because of the controlled environmental and social context. In the present study we used a similar approach, examining assaults by offenders in a forensic hospital. A finding similar to Sorensen et al. that IPV offenders commit fewer institutional assaults would add to evidence that, across different samples and methods, criminal propensity is lower among IPV offenders than other offenders.

\section{ACEs and Criminal Propensity}

It has been suggested that ACEs might increase criminal propensity directly, through a mechanism related to trauma (e.g., Greene, Ford, Wakefield, \& Barry, 2014) or indirectly by disrupting intimacy development thus interfering with familial relationships (e.g., Levenson \& Socia, 2015). Evidence reviewed here, however, suggests that IPV offenders have higher ACEs but lower criminal propensity than other offenders on average, which raises the question as to 
whether ACEs are a criminogenic treatment need, at least among IPV offenders. There are few studies of both ACEs and criminal propensity among IPV offenders to address this question.

The first study of criminal propensity and IPV in relation to an adverse childhood event involved 75 offenders awaiting trial, 21 of whom reported perpetrating IPV (Swogger, Walsh, Kosson, Cashman-Brown, \& Caine, 2012). Self-reported childhood physical abuse was related to IPV and to facets of psychopathy, measured by the PCL-R. Furthermore, lifestyle traits of psychopathy, but not antisociality or violent behavior, moderated the association of abuse and IPV such that self-reported abuse and IPV were most strongly associated among the most psychopathic offenders. Swogger and colleagues suggested early intervention with abused children who exhibit impulsivity to reduce their risk of violence in later relationships. Another study examined the range of ACEs among 61 male and female forensic outpatients receiving court-ordered or voluntary IPV perpetrator treatment or who had a criminal history of IPV (Henrichs et al., 2015). They compared intake assessment reports of childhood abuse from these patients with those from 58 forensic outpatients receiving treatment for violence against nonintimate partners. Childhood physical abuse, but not psychological or sexual abuse, was more common among IPV offenders than offenders with non-IPV violence. There was little difference between the groups in their general criminal history. Their study was the first to examine the generalizability of ACEs among IPV offenders in a specifically forensic sample, and it suggests that, although IPV offenders may have higher rates of childhood abuse than others, this adverse childhood event does not necessarily confer greater criminal propensity on IPV offenders.

There is potential value in extending the study of ACEs among IPV offenders in clinical samples. Men who admit perpetrating IPV report a higher rate of serious mental illness than others in the community (e.g., Lipsky, Caetano, \& Roy-Byrne, 2011). Emerging evidence 
suggests that ACEs are prevalent among forensic inpatients (Roters, Marshall, Keating, \& Ball, 2015), and there is increasing expectation that traumatic childhood events require treatment among IPV offenders (e.g., Semiatin, Torres, LaMotte, Portnoy, \& Murphy, 2016) which is likely to be accepted by forensic institutions that tend to emphasize treatment of psychiatric needs and embrace trauma-informed care. If ACEs are related to criminal propensity among IPV offenders, they could be considered a treatment priority according to the Risk-Need-Responsivity (RNR) principles of effective offender service (Andrews \& Bonta, 2010). If there is no replicable association between ACEs and criminal propensity among IPV offenders, though, it is possible that ACEs are not criminogenic needs for IPV offenders, and other treatment needs should be prioritized in order to adhere to the RNR model. Thus, there is a need for further investigation of the association between ACEs and criminal propensity in this population.

In summary, community and institutional studies of IPV offenders have typically found this subgroup to exhibit on average less general antisociality and to have lower actuarial risk for subsequent violent criminal behavior than other violent offenders. Together with evidence that ACE prevalence is elevated among IPV offenders, these findings raise the question of whether ACEs would be associated with criminal propensity among IPV offenders. The present study addressed this question in a sample of IPV and other offenders assessed in a forensic institution.

\section{ACEs, IPV, and Measuring Intimate Partner Relationship History}

There is a methodological issue in research to date that raises the need to control for intimate partner relationship history. Research comparing men who commit IPV with men assaulting non-partners has a potential confound in that having had a marital relationship is a protective factor for violence risk (e.g., Harris et al., 2015). Having had multiple short-term intimate relationships, on the other hand, is a characteristic of psychopathy (Hare, 2003). 
Previous research comparing IPV offenders with other offenders controlled for similar criminal history (e.g., Henrichs et al., 2015; Sorenson et al., 2014) but not for intimate relationship history, so the differences reported could be in some way attributable to factors related to having had an intimate partner. In the present study, we recorded whether offenders had ever been married, cohabited with an intimate partner, or had a dating relationship and we controlled for this intimate partner relationship history in our tests of the main hypotheses.

\section{The Present Study}

Offenders were drawn from a population of men admitted to a secure forensic psychiatric institution. Although this sample was selected for convenience and had a higher expected prevalence of mental illness than previous samples, it allowed us to examine institutional behavior and also garner information from detailed medical records, psychosocial histories, and information from other sources including some from childhood. These records captured information about not only verbal, physical, and sexual abuse and neglect experiences, but also parental characteristics including separation, domestic violence, substance use, mental illness, and criminal history, permitting a more complete measure of ACEs than previously reported among IPV offenders. It also allowed us to examine well-documented measures of criminal propensity, including psychopathy, actuarial risk of recidivism, violent and nonviolent criminal history, as well as assaults in the institution, among IPV offenders and other offender groups.

We compared IPV offenders with offenders who committed violence against nonintimate partners (non-IPV) as in some previous research (e.g., Henrichs et al., 2015; Swogger et al., 2007). In addition we identified nonviolent offenders, who had no current or prior violent charges. This third group extended previous research from its existing focus on violent and sexual offenders and allowed us to examine whether ACEs are equally prevalent among 
nonviolent offenders. Using an institutional sample meant we anticipated a higher rate of mental disorder than one would expect in a community sample, but a rate perhaps more comparable to incarcerated offenders as mental illness is prevalent in correctional institutions (e.g., Prins, 2014). This sample also allowed the present study to build on recent work on institutional assaults by IPV offenders (Sorensen et al., 2015). With respect to intimate relationship history, the anticipated moderate prevalence permitted use of this variable as a covariate, a methodological improvement on previous research that did not control for such history.

\section{Research Questions and Associated Hypotheses}

1. Will IPV offenders have more ACEs than non-IPV offenders and nonviolent offenders? We expect, based on Henrichs et al. (2015) that ACE scores will be higher among IPV than non-IPV offenders. If this outcome is replicated, it would bear implications for understanding the effect of ACEs, consistent with the suggestion that the experiences disrupt how people behave in intimate relationships.

2. Will IPV offenders exhibit lower criminal propensity than non-IPV offenders and nonviolent offenders? We expect, based on Theobald et al. (2015) and Hilton et al. (2001), that IPV offenders will have less violent and nonviolent criminal history, psychopathy, and actuarial risk of violent recidivism. Based on Sorensen et al. (2015), we also expect that IPV offenders will commit fewer institutional assaults. If this outcome is obtained, it would bear implications for whether IPV offenders should be given lower priority for offender intervention in accordance with the risk principle of the RNR model.

3. Because there is some evidence that ACEs are higher among IPV offenders, yet IPV offenders appear to score lower on at least some facets of psychopathy and are at lower actuarial risk of violent recidivism than other offenders, we further ask whether ACEs are associated with 
criminal propensity among IPV offenders. An association would provide evidence for the robustness of the association between ACEs and general antisociality, which would be consistent with targeting ACEs as a criminogenic need among IPV offenders.

\section{Method}

The present study was conducted as part of a longitudinal study of all forensic patients admitted to a secure psychiatric hospital in Canada for assessment during the four-year period from January 2009 to December 2012. We used an archival method and all data were collected retrospectively. Shortly after each patient's admission, we coded information from medical records that included details of criminal offenses leading to admission (index offense), criminal history, childhood and adult psychosocial history, and initial adjustment to the institution. For a subset of the sample we returned to the medical records after patients' admission anniversary date to code data regarding events during the hospital stay, masked to the main data collection. We identified IPV offenders from their index offense or prior criminal charges. The non-IPV comparison group consisted of men whose index offense was violent, but who never had charges for an offense against their partner. A third, nonviolent group was also identified.

\section{Sample}

There were 638 men admitted in the study period. Of this sample, $99(16 \%)$ had been charged for a violent offense against a current or previous marital, cohabiting, or dating partner, either during their index offense $(n=7)$, or during a criminal offense at any time prior to this admission $(n=92)$. These 99 men constituted the IPV group. Of the remainder, 315 had a violent charge in their index offense and no history of IPV, and 223 had nonviolent charges and no history of IPV. We excluded 82 offenders with a violent index charge who had an undetermined intimate relationship history based on available records, leaving 233 non-IPV 
offenders. We excluded 50 offenders with only nonviolent index charges who had an undetermined intimate relationship history and a further 70 with prior violent charges, leaving 103 nonviolent offenders. Results are therefore reported on 99 IPV, 233 non-IPV, and 103 nonviolent offenders, for a total sample of 435 .

\section{Procedure}

The study was reviewed and approved by the authors' institutional research ethics board. Information pertaining to criminal offenses leading to admission, criminal history (e.g., preindex charges), childhood psychosocial history (e.g., adverse childhood experiences) and adult psychosocial history (e.g., psychopathy), and actuarial risk of violence were coded from medical records within three to six months of patients' admission to the assessment unit. These variables were coded from information in the psychosocial history report prepared by clinical social workers and psychological reports prepared by clinical forensic psychologists, the criminal record containing all charges and convictions, and other relevant information accompanying the medical record. Most cases $(471,93 \%)$ were coded by one of two experienced researchers who initially coded cases by consensus then commenced the bulk of the coding independently. Ten cases were used to assess masked inter-rater reliability (described below). Some cases $(34,7 \%)$ were coded by a trainee researcher who also achieved reliability with an experienced coder.

\section{Measures}

ACEs were captured in nine separate variables, which were coded as present based on evidence documented in the medical record, including but not limited to the psychosocial report completed by one of four social workers on the unit who gathered information from other sources and usually consulted with the patient and/or family informants. The psychosocial report is not a standardized tool but a method of reporting on current offense details, family history, 
childhood background, criminal history, adult relationships, and other information. Childhood abuse, neglect, or witnessed violence were coded as missing and subsequently treated as not present if the only informant was an offender reported to be a poor historian or to contradict himself. Abuse-related variables included experiencing verbal abuse (nonphysical, psychological abuse), physical abuse, sexual abuse, neglect of basic emotional or physical needs, or witnessing the physical or sexual abuse of the mother. Additional ACE variables included separation from biological father before the age of 16 , mother or father having a mental illness, mother or father having problematic alcohol or drug use, or mother or father having a criminal record. We calculated an ACE score by adding 1 point for each one of these events coded as present. The absence of evidence for any of the component items was scored 0 . The ACE score therefore had a possible range of 0-9.

Intimate partner relationship history was coded as present if any of these conditions were met at the time of admission: was married and living with partner, not married but living with partner, previously married now living apart, dating and never lived together, was dating now split up. It was coded as absent if there was evidence that the offender was single and had not dated or lived with a partner.

Criminal Propensity. Criminal propensity was operationalized in terms of violent and nonviolent criminal history, psychopathy, and actuarial risk for violent recidivism. We coded criminal history from the criminal record of all charges and convictions incurred in Canada, drawn from the Canadian Police Information Centre and documented on the medical record at the time of admission. We coded extent of criminal history separately for violent and nonviolent offenses prior to the most recent offense, using the Cormier-Lang Criminal History Score (Harris et al., 2015). This measure assigns a numeric value for the severity of each charge multiplied by 
the number of counts of that charge, which is then summed across all charges. Some offenders had very lengthy criminal records, so we coded violent criminal history up to a score of 20 (the $75^{\text {th }}$ percentile in prior data collection) and nonviolent criminal history up to 3 (the maximum needed to score this item for the Violence Risk Appraisal Guide [VRAG], described below).

Psychopathy was measured using the PCL-R (Hare, 2003). The PCL-R is a standard tool for assessing psychopathy using documentation and offender interview, with well-established psychometric properties including replicable factor structures, test-retest reliability, evidence for good inter-rater reliability, and convergent, divergent, and predictive validity, as well as general consistency across offender ethnicities (e.g., Harris, Rice, \& Cormier, 2013; Salekin, Rogers, \& Sewell, 1996; Sullivan, Abramowitz, Lopez, Kosson, 2006). The PCL-R is a restricted psychological test and the assessments used in this study were conducted by one of three registered psychologists trained in use of the tool, based on file review and offender interview whenever possible. The PCL-R total score has a possible range of $0-40$. We did not score the PCL-R for this research but used the total score documented on the medical record by the clinical psychologist who completed the assessment. The clinician-scored PCL-R score was documented for 119 cases in the present study. Missing data was not at random but associated with shorter length of stay, MCAR $\chi^{2}(d f=2)=52.51, p<.001$. We did not impute data but report results for PCL-R score based on complete cases for this variable.

We scored the VRAG (Harris, Rice, \& Quinsey, 1993; Harris et al., 2015) using information from the psychosocial history and criminal record documented on the medical record. The VRAG is an actuarial instrument for assessing the risk of violent recidivism. Its predictive accuracy is well established and the average effect size (measured by the receiver operating characteristic area under the curve) is over .71 across studies with a range of 
populations and researchers (e.g., Harris, Rice, \& Quinsey, 2010) and in follow ups of 6 months to 49 years (Rice, Harris, \& Lang, 2013). Its 12 component items (Table 2) are weighted such that the VRAG total score has a possible range of -22 to +33 and prorating can be used for up to four missing items. PCL-R score is the highest weighted item, and where it was missing we substituted the Child and Adolescent Taxon Scale (CATS; according to instructions in Harris et al., 2015) which consists of a score based on the following items: elementary school maladjustment, teenage alcohol problem, childhood aggression problem, childhood conduct problems before age 15, ever suspended or expelled from school, arrested under the age of 16, parental alcoholism, separated from either biological parent before age 16 . We prorated the CATS out of 40 then used the PCL-R based weights.

We also measured institutional assaults defined as forceful physical contact with another person, in the subset of 273 cases for which we coded institutional behavior up to the first year. Most were discharged from the institution after assessment and only $13 \%$ remained a full year.

\section{Inter-rater Reliability}

Variables reported in this study yielded correlation coefficients of ICC (mixed model, absolute agreement, single measure) $>.75$, including .87 for the sum of the ACE items and perfect agreement on separation from biological father, parental mental illness, parental alcohol or drug use problem, parental crime, Cormier-Lang scores for violent charges and nonviolent charges, and whether the offender had an intimate partner relationship.

\section{Analytical Plan}

We computed descriptive statistics for each of the dependent measures and each offender group, and tested whether there were systematic differences between groups in the prevalence of major mental illness. We tested hypothesis 1, that IPV offenders would have more ACEs than 
others, using chi squared statistics for categorical measures (individual events) and one way analysis of variance (ANOVA) of group means for the continuous ACE score with intimate partner relationship as a covariate. We tested hypothesis 2, that IPV offenders would show more criminal propensity than others, also using ANOVA for continuous data (PCL-R, VRAG, violent and nonviolent criminal history score and number of assaults). We examined the $95 \%$ confidence interval (CI) around group means to test whether the IPV group significantly differed from the comparison groups; between-group statistical differences were inferred when one point estimate fell outside the $95 \% \mathrm{CI}$ of another. We also examined the receiver operating characteristic area under the curve (AUC), a statistic more robust than correlation coefficients to base rate variations (e.g., Babchishin \& Helmus, 2015) to test whether independent variables discriminated IPV offenders from remaining offenders. We tested hypothesis 3, that ACEs would be associated with criminal propensity among IPV offenders, using a series of linear regression analyses conducted separately for each criminal propensity measure (Cormier-Lang scores, PCL-R, VRAG, and institutional assaults) in the subsample of IPV offenders.

\section{Results}

The 435 male offenders had a mean age at their index offense of 34.31 years $(S D=$ 12.51). They had a mean of 11 years of education $(S D=3.13)$ and $256(59 \%)$ were on government benefits prior to admission. The most serious index charges were common assault $(n=113,26 \%)$, threatening $(n=46,9 \%)$, assault with a weapon $(n=27,6 \%)$ or other charges ranging from first degree murder $(n=10,2 \%)$ to causing disturbance $(n=2,1 \%)$. Offenders' country of birth indicated a diverse sample, with one third $(n=141,32 \%)$ born outside Canada. The most common other country of birth was Jamaica $(n=16,4 \%)$, followed by China $(n=7$, 
$2 \%)$ and the Philippines $(n=6,1 \%)$; in total nearly 60 countries of birth were represented. The mean ACE score was $1.71(S D=1.75)$ with a range of $0-8$ and internal reliability of $\alpha=.68$.

Most had a primary diagnosis of schizophrenia $(175,40 \%)$ with no significant difference between offender groups $(31 \%-51 \%), \chi^{2}(1,434)=4.10, p=.129$. Remaining primary diagnoses were other psychosis $(87,20 \%)$, mood disorder $(46,11 \%)$, personality disorder $(41$, $9 \%)$, substance related diagnosis $(29,7 \%)$, paraphilia $(4,1 \%)$, or other diagnosis (e.g., developmental delay, ADHD; 45, 10\%). A further eight (2\%) had no psychiatric diagnosis. Of the subset of 241 cases included in the anniversary date coding, $63(26 \%)$ had been found not criminally responsible (NCR) and 63 (26\%), unfit to stand trial. Others were released to court and were not returned to hospital afterwards; in our experience this may due to their criminal conviction in many cases, but their disposition was not documented in the medical record. Based on the subset, NCR findings differed significantly between offender groups, the non-IPV group being most likely to be found NCR (35\%) compared with IPV offenders (25\%) and nonviolent offenders $(15 \%), \chi^{2}(2,240)=15.43, p<.001$. Further analyses were restricted to ACEs, IPV offending and criminal propensity without controlling for mental disorder variables.

\section{Hypothesis 1: IPV offenders will have more ACEs than non-IPV offenders}

There was an overall effect of offender group on ACE score, $F(2,431)=3.25, p=.004$ and no significant effect of intimate history $F(1,431)=2.42, p=.121$. Three individual ACE items yielded overall group differences: childhood experience of verbal abuse, childhood experience of physical abuse, and parental substance abuse. Higher ACE score was not significantly associated with IPV offender group membership, AUC $=.563(S E=.034), 95 \%$ CI $=[.497, .629]$. IPV offenders had significantly higher ACE scores than nonviolent offenders as inferred from CIs (Table 1) and post-hoc contrast, IPV vs. nonviolent offenders $p=.012$, but 
were not significantly different from non-IPV offenders. Furthermore, item by item comparisons reveal that the IPV group was more similar to non-IPV than either was to nonviolent offenders, except for a higher rate of parental substance abuse among IPV offenders than either non-IPV or nonviolent offenders (Table 1). These results do not support the hypothesis to the extent that IPV offenders did not have more ACEs than non-IPV offenders specifically.

\section{Hypothesis 2: IPV offenders will exhibit lower criminal propensity than non-IPV and} nonviolent offenders, as measured by violent and nonviolent criminal history, psychopathy, and actuarial risk of violent recidivism

There was an overall effect of offender group on both criminal history scores, violent $F$ $(2,250)=8.90, p=.003$, nonviolent $F(2,306)=14.05, p<.001$. There were no significant effects of intimate partner history, $F(1,307)=1.70, p=.193$, and $F(1,306)=.036, p=.551$. Contrary to the hypothesis, IPV offenders had more extensive pre-index violent criminal history than non-IPV offenders as inferred from CIs and post-hoc contrast test, $p<.001$, and more extensive nonviolent history than both other groups, both $p<.001$. Higher criminal history scores were significantly associated with IPV offender group membership, violent AUC $=.702$ $(S E=.032), 95 \% \mathrm{CI}=[.638, .766]$, nonviolent $\mathrm{AUC}=.644(S E=.034), 95 \% \mathrm{CI}=[.578, .771]$.

There was an overall effect of offender group on clinician-scored PCL-R, $F(2,90)=$ $5.53, p=.005$ and no significant effect of intimate partner history, $F(1,90)=0.90, p=.346$. Contrary to the hypothesis, the IPV group scored higher than both other groups as inferred from CIs, and a significant post-hoc contrast between IPV nonviolent offenders, $p=.002$. Higher PCL-R score was significantly associated with IPV offender group membership, AUC $=.720$ $(S E=.054), 95 \% \mathrm{CI}=[.614, .827]$. 
There was no overall effect of offender group scores on the VRAG, $F(1,393)=2.71, p=$ $.068, \mathrm{AUC}=.555(S E=.034), 95 \% \mathrm{CI}=[.487, .623]$. There was a significant effect of intimate partner relationship, $F(1,393)=6.08, p=.014$. In post-hoc analysis, relationship history was associated with lower VRAG scores, $r(463)=-.109, p=.030$. There was no overall effect of offender group on the number of institutional assaults, $F(1,235)=2.26, p=.106$, AUC $=.418$ $(\mathrm{SE}=.044), 95 \% \mathrm{CI}=[.332, .504]$, and no significant effect of intimate relationship history, $F$ $(1,235)=0.16, p=.695$. The offender group effect appeared attributable to the non-IPV group tending to commit most assaults but this trend was not significant as inferred from CIs or in posthoc contrast tests, $p s=.078-.877$. These results do not support our hypothesis that IPV offenders will have lower criminal propensity than others. The hypothesis was contradicted for criminal history and psychopathy.

\section{Hypothesis 3: ACEs and Criminal Propensity among IPV Offenders}

Overall, higher ACE scores were associated with more criminal propensity on all measures except institutional assaults, as shown by correlation coefficients in Table 1. Among IPV offenders, linear regression analyses conducted separately for each criminal propensity measure yielded a significant model for VRAG score only, $F(1,88)=14.41, r(89)=.375, p<$ .001. No association with ACEs was found among IPV offenders for other criminal propensity measures: violent criminal history, $F=2.19, r(78)=.166, p=.143$, nonviolent criminal history, $F(1,77)=1.71, r(78)=.147, p=.195$, PCL-R, $F(1,19)=0.00, r(20)=.004, p=.985$, assaults, $F(1,42)=2.41, r(43)=.233, p=.128$. These results provide inconsistent evidence for the hypothesis that ACEs will be directly associated with criminal propensity among IPV offenders.

\section{Discussion}


We compared 99 IPV offenders with other violent and nonviolent male offenders undergoing forensic assessment in a psychiatric hospital during the same time period. Consistent with hypothesis 1, ACE scores differed among the three offender groups, with IPV offenders having the highest ACE scores. Our study was conducted with a forensic inpatient sample, but this result is similar to previous research finding that ACEs are associated with IPV perpetration among non-offenders (e.g., Whitfield et al., 2003) or offenders under community supervision (Swogger et al., 2012). Specific adverse events for which this pattern was statistically significant were exposure to verbal and physical abuse and parental substance abuse. IPV offenders had higher ACE scores than nonviolent offenders but were similar to non-IPV offenders on the total score and most individual adverse events. We conclude that this evidence supports the generalizability of the prevalence of ACEs among IPV offenders, but there is insufficient evidence that ACEs distinguish men who commit IPV from other violent offenders. Thus this study was unable to support the suggestion that ACEs disrupt intimate relationships specifically. Contrary to hypothesis 2, IPV offenders exhibited more criminal propensity than other offenders on measures of criminal history and psychopathy. They had significantly more violent criminal history than non-IPV offenders and higher PCL-R scores than non-IPV and nonviolent offenders. Theobald et al. (2015) reported lower psychopathy among IPV perpetrators compared with men who committed other violence (e.g., Theobald et al., 2015); however, their study used different criteria for IPV (self-report) and other violence. In contrast, we used the same criteria for IPV as for other violence (i.e., criminal charges) and found that IPV offenders exhibited more psychopathic traits than men with non-IPV or nonviolent charges. Theobald et al. used a short version of the PCL-R scored by researchers, whereas in our study psychopathy was measured using the PCL-R as scored by clinicians, but we found a similar pattern of association between 
ACE scores and criminal propensity when we used violent and nonviolent criminal history measures scored by researchers as the criminal propensity index, lending some confidence to this finding. Theobald et al. also used a community cohort, whereas we sampled from a forensic institution. It appears that, among offenders court-ordered to psychiatric assessment, those who have assaulted an intimate partner are relatively prone to criminal activity and psychopathy. The results obtained for the actuarial measure of violence risk and for violence in the institution were different but also contradicted hypothesis 2 . An overall group difference in the rate of institutional assaults appeared attributable to more assaults by the non-IPV offenders. This finding indicates some stability of Sorensen and colleagues' (2015) report that IPV homicide offenders committed fewer prison assaults than non-IPV homicide offenders.

Consistent with hypothesis 3 and with previous research indicating an association of ACEs with criminal offending, ACEs contributed to all models of criminal propensity except for institutional assaults in the total sample of all offenders. However, among IPV offenders specifically this association held only for actuarial risk of violent recidivism; two items overlap in the VRAG and ACE scores -- parental alcoholism and separation from parents -- possibly explaining this result. This finding provides limited support for ACEs being related to criminal propensity among IPV offenders, although the small subsample and the restricted range of ACE scores compared with the total sample could have contributed to lack of power and consequent null findings. Further study of the association of ACEs and criminal propensity in a larger sample of IPV offenders may be advisable before conclusions can be drawn about whether and how ACEs contribute to IPV offending per se.

We made no hypothesis about intimate relationship history but controlled for it because of a potential confound in previous research examining associations among ACEs and offending 
patterns among IPV offenders. There was no significant effect of having had an intimate partner on either ACEs or criminal propensity measures, except for risk of violent recidivism, whereby having an intimate relationship history was associated with lower VRAG scores, which is partly attributable to marital history being a VRAG item. We conclude from this overall pattern of results that intimate relationship history does not confound previous research findings.

\section{Limitations}

The present study was based on archival data and we did not solicit self-reports from offenders. The medical records included psychosocial data compiled by clinicians as well as official criminal records and captured offenders' self-reports through clinical interviews. Nevertheless, this archival method likely underestimates ACEs relative to self-report estimates (notwithstanding low test-retest reliability of retrospective reports of childhood abuse; e.g., Fergusson et al., 2008a), and limits the comparability of our findings to other studies that did use self-report. In addition, the PCL-R score was missing from the record in most cases, limiting our assessment of criminal propensity using this key measure.

Our sample consisted entirely of offenders undergoing psychiatric assessment in a forensic hospital, many of whom were found unfit to stand trial or not criminally responsible. Being found NCR was not common among IPV offenders and was highest among the non-IPV group, perhaps contributing to their rate of institutional assaults. Mental illness was prevalent in the sample but did not differ significantly between groups. A strength of this sample is that we were able to replicate, in a clinical sample with relatively serious offending history, the finding from community-based research that ACEs are more common among IPV offenders than other offenders. It allowed us to demonstrate the robustness of this phenomenon across samples that differed on characteristics such as mental disorder and severity of offense history and draw some 
preliminary inferences about clinical intervention. Also, because the institution served a large and ethnically diverse region in Canada, this diversity was reflected in our sample. We also note that, although psychiatric patients have been found to assault partners and other family members at a higher rate than the general population (e.g., Labrum \& Solomon, 2015), the proportion of our offender sample with an IPV history, about one in five, was similar to that reported by Swogger et al. (2012) in their sample of men in a community supervision program. Our sample cannot, however, be assumed to represent either community samples of IPV offenders or offender populations such as have been sampled in previous research in this field, as most were clinically diagnosed with a mental disorder.

Our definition of IPV offenders included current and previous offenses, which limits the comparability of our findings to studies that selected IPV offenders by other means (e.g., current offenses, lifetime self-report). For example, Hilton et al. (2001) identified IPV offenders using official records but used only the index offense to do so, whereas we examined pre-index offenses as well. Nevertheless, our inclusive definition is more naturalistic than restriction to current offenses, and may better reflect IPV offenders who commit other offenses as well.

We had a small sample for our tests of ACE associations within IPV offenders, especially for the clinician-scored PCL-R. Also with respect to psychopathy, we did not have information on the different factors or facets of the PCL-R. There is some potential overlap of the PCL-R and VRAG; however, due to missing PCL-R scores this item was often substituted with the CATS, which covers only behavioral aspects of antisociality. Previous research has found that only certain facets of psychopathy distinguish IPV offenders (e.g., Swogger et al., 2007). Our findings might be primarily influenced by the facet reflecting criminal history. Future research should explore the relation of the full range of ACEs to multiple facets of the PCL-R. 


\section{Implications for Research}

Previous research found that men who commit IPV scored lower on measures of psychopathy and actuarial risk, unlike the present study. This difference might be attributable to different data collection and sampling. For example, Theobald et al. (2015) measured IPV using self report, whereas we obtained information from criminal records, psychosocial histories, and other reports contained in the medical record. As another example, Swogger et al. (2007) compared features of psychopathy among incarcerated men who all had antisocial personality features, whereas less than $10 \%$ of our sample was diagnosed with any personality disorder. Some men not classified as IPV offenders in our study might have committed IPV without it coming to the attention of the criminal justice system, or IPV might have resulted in charges not identifiable as IPV from the available information. Because IPV offenders often commit different types of violent and nonviolent offenses over their career (e.g., Hilton \& Eke, 2016; Piquero, Brame, Fagan, \& Moffitt, 2006) such that using a single offense might not capture all IPV offenders, the present study might have correctly identified more IPV offenders. We perhaps also identified more generally violent offenders, thought to be a subtype of IPV offending responsible for relatively severe and repeated violence against their partners (e.g., Cunha \& Gonçalves, 2013). The generally antisocial IPV offender subtype appears largely consistent over time (e.g., Holtzworth-Munroe, Meehan, Herron, Rehman, \& Stuart, 2003) and across samples, including psychiatric inpatients (e.g., Walsh et al., 2010). Future research might examine whether the link between ACEs and IPV differs across batterer subtypes.

Previous research controling for offence type found that IPV offenders presented with better institutional behavior than other offenders (Sorensen et al., 2015), similar to the present study, at least in comparison to non-IPV offenders. Sorensen et al. studied only homicide 
offenders, which might be a limitation because victim death can be a protective factor for violent recidivism (e.g., Harris et al., 2015). We used a sample with less serious violent offenses and controlled for intimate relationship history, and found that non-IPV offenders were most likely to commit assaults. However, Sorenson and colleagues' study included not only assaults but a broad range of rule violations, and had a higher prevalence of observed assaults than our study, resulting in greater sensitivity to between-group differences. Further research into the association of ACEs with institutional violations would benefit from a similar methodological approach. This recommendation is also supported by Swogger and colleagues' (2007) finding that affective and lifestyle facets of psychopathy exhibited opposite associations with IPV offending, indicating that criminal propensity among IPV offenders relative to others is more nuanced than perhaps we were able to capture in the present study.

\section{Implications for Practice}

We found that ACE score was associated with IPV offending and that IPV offenders scored higher on some measures of criminal propensity. A clear indication that ACEs contribute to criminal propensity among IPV offenders, however, remains to be found. As a result, there are no clear implications supporting the treatment of ACEs among IPV offenders.

The most prevalent adverse events for this group were separation from father and parental substance abuse. This substance abuse finding contrasts with that reported by Fergusson et al. (2008b). However, the apparent association of ACEs and violence risk could be attributable to overlapping content. Parental substance abuse is included in the VRAG item "history of alcohol problems" and separation relates to the item "lived with both biological parents to age 16" (Harris et al., 2015, p. 285). The association of parental substance abuse, which was highest among IPV offenders in our sample, with actuarial risk of recidivism offers 
more support for assessing and treating substance abuse among IPV offenders (e.g., Radatz \& Wright, 2015) than for attending to trauma-focused treatment.

According to Andrews and Bonta (2010), interventions to help offenders deal with a history of trauma and abuse are considered not "compatible" with the principles of effective intervention (p. 509). Furthermore, the mechanism of the association between ACEs and overall criminal propensity, be it trauma, interference of intimacy development, or antisocial development is not well understood. On the other hand, clinical interventions for offenders have incorporated trauma treatment and reported success in reducing symptoms (Messina, Calhoun, \& Braithwaite, 2014) and IPV recidivism (e.g., Crane et al., 2013). Future research could evaluate the incremental benefit of assessing and treating ACEs and related trauma on IPV offending.

IPV offenders on the whole were not observed to commit more institutional violence than non-IPV and nonviolent offenders; consequently, it seems IPV offenders do not appear to merit higher priority for institutional security compared with other offenders on the basis of their particular offense history. This conclusion is offered with caution as institutional assaults were based on only a subset of the sample and most offenders stayed less than a year. Meanwhile, they scored no higher than other violent offenders on actuarial risk of recidivism, so do not appear to merit higher priority for offense-reduction intervention overall compared with other offenders in this population, although evidence of their greater extent and diversity of criminal offending (indicated by higher PCL-R scores and violent and nonviolent criminal history) suggests that treatment should focus on criminogenic needs related to general antisocial behavior. 


\section{References}

Andrews, D. A., \& Bonta, J. (2010). The psychology of criminal conduct (5th ed.). Newark, NJ: Lexis Nexis.

Bellis, M. A., Lowey, H., Leckenby, N., Hughes,K., \& Harrison, D. (2013). Adverse childhood experiences: Retrospective study to determine their impact on adult health behaviours and health outcomes in a UK population. Journal of Public Health, 36, 81-91. doi:10.1093/pubmed/fdt038

Babchishin, K. M., \& Helmus, M. L. (2015). The influence of base rates on correlations: An evaluation of proposed alternative effect sizes with real-world data. Advance online publication. Behavior Research Methods. doi:10.3758/s13428-015-0627-7

Björkenstam, E., Hjern, A., Mittendorfer-Rutz, E., Vinnerljung, B., Hallqvist, J., \& Ljung, R. (2013). Multi-exposure and clustering of adverse childhood experiences, socioeconomic differences and psychotropic medication in young adults. PLOS ONE, 8, 1-9.

Capaldi, D. M., Knoble, N. B., Shortt, J. W., \& Kim, H. J. (2012). A Systematic review of risk factors for intimate partner violence. Partner Abuse, 3, 231-280. doi:10.1891/19466560.3 .2 .231

Corvo, K., \& Carpenter, E. H. (2000). Effects of parental substance abuse on current levels of domestic violence: A possible elaboration of intergenerational transmission processes. Journal of Family Violence, 15, 123-135.

Crane, C. A, Oberleitner, L. M. S., \& Easton, C. (2013). Sub-clinical trauma in the treatment of partner violent offenders with substance dependence. Advances in Dual Diagnosis, 6, 513. doi:10.1108/17570971311308980 
Cunha, O., \& Gonçalves, R. A. (2013). Intimate partner violence offenders: Generating a databased typology of batterers and implications for treatment. European Journal of Psychology Applied to Legal Context, 5, 131-139. doi:10.5093/ejpalc2013a2

Dong, M., Anda, R. F., Felitti, V. J., Dube, S. R., Williamson, D. F., Thompson, T. J., Loo, C. M., \& Giles, W. H. (2004). The interrelatedness of multiple forms of childhood abuse, neglect, and household dysfunction. Child Abuse and Neglect, 28, 771-784. doi:10.1016/j.chiabu.2004.01.008

Dube S. R., Anda, R. F., Felitti, V. J., Edwards, V. J., \& Williamson D. F. (2002). Exposure to abuse, neglect, and household dysfunction among adults who witnessed intimate partner violence as children: Implications for health and social services. Violence and Victims, $17,3-17$.

Felitti, V. J., Anda, R. F., Nordenberg, D., Williamson, D. F., Spitz, A. M., Edwards, V., Koss, M. P., \& Marks, J. S. (1998). Relationship of childhood abuse and household dysfunction to many of the leading causes of death in adults: The Adverse Childhood Experiences (ACE) Study. American Journal of Preventive Medicine, 14, 245-258.

Fergusson, D. M., Boden, J. M., \& Horwood, L. J. (2008)a. Exposure to childhood sexual and physical abuse and adjustment in early adulthood. Child Abuse \& Neglect, 32, 607-619. doi: 10.1016/j.chiabu.2006.12.018

Fergusson, D. M., Boden, J. M., \& Horwood, L. J. (2008)b. Developmental antecedents of interpartner violence in a New Zealand birth cohort. Journal of Family Violence, 23, 737-753. doi: 10.1007/s10896-008-9199-y 
Finkelhor, D. Shattuck, A., Turner, H., \& Hamby, S. (2013). Improving the Adverse Childhood Experiences Study Scale. Journal of the American Medical Association: Pediatrics, 167, 70-75. doi:10.1001/jamapediatrics.

Greene, C. A., Ford, J. D., Wakefield, D. B., \& Barry, L. C. (2014). Posttraumatic stress mediates the relationship between childhood victimization and current mental health burden in newly incarcerated adults. Child Abuse and Neglect, 38, 1569-1580. doi: 10.1016/j.chiabu.2014.07.006

Hare, R. D. (2003). Manual for the Revised Psychopathy Checklist, 2nd ed. Toronto, ON: MultiHealth Systems.

Harris, G. T., Rice, M. E., \& Cormier, C. (2013). Research and Clinical Scoring of the Psychopathy Checklist can show good Agreement. Criminal Justice and Behavior, 40, 1349-1362. doi: 10.1177/0093854813492959

Harris, G. T., Rice, M. E., \& Quinsey, V. L (2010). Allegiance or Fidelity? A Clarifying Reply. Clinical Psychology: Science and Practice, 17, 82-89.

Harris, G.T., Rice, M.E., \& Quinsey, V.L. (1993). Violent recidivism of mentally disordered offenders: The development of a statistical prediction instrument. Criminal Justice and Behavior, 20, 315-335.

Henrichs, J., Bogaerts, S., Sijtsema, J., \& Klerx-van Mierlo, F. (2015). Intimate partner violence perpetrators in a forensic psychiatric outpatient setting: Criminal history, psychopathology, and victimization. Journal of Interpersonal Violence, 30, 2109-2128. doi: $10.1177 / 0886260514552272$. 
Hilton, N. Z. \& Eke, A. W. (2016). Nonspecialization of criminal careers among intimate partner violence offenders. Criminal Justice and Behavior. Advance online publication. doi: $10.1177 / 0093854816637886$

Hilton, N. Z., Harris, G. T., \& Rice, M. E. (2001). Predicting violent recidivism by serious wife assaulters. Journal of Interpersonal Violence, 16, 408-423. doi:10.1177/ 088626001016005002

Holtzworth-Munroe, A., Meehan, J. C., Herron, K., Rehman, U., \& Stuart, G. L. (2003). Do subtypes of maritally violent men continue to differ over time? Journal of Consulting and Clinical Psychology, 71, 728-740.

Jespersen, A. F., Lalumière, M. L., \& Seto, M. C. (2009). Sexual abuse history among adult sex offenders and non-sex offenders: A meta-analysis. Child Abuse \& Neglect, 33, 179-192. doi:10.1016/j.chiabu.2008.07.004

Kelly-Irving, M., Lepage, B., Dedieu, D., Bartley, M., Blane, D., Grosclaude, P., Lang, T., \& Delpierre, C. (2013). Adverse childhood experiences and premature all-cause mortality. European Journal of Epidemiology, 28, 721-734. doi:10.1007/s10654-013-9832-9

Kroner, D. G., Mills, J. F., \& Reddon, J. R. (2005). A coffee can, factor analysis, and prediction of antisocial behavior: The structure of criminal risk. International Journal of Law and Psychiatry, 28, 360-374.

Krupp, D. B., Sewall, L. A., Lalumière, M. L., Sherriff, C. \& Harris, G. T. (2013). Psychopathy, adaptation, and disorder. Frontiers in Psychology, 27 March 2013, doi:10.3389/fpsyg.2013.00139

Lipsky, S., Caetano, R., \& Roy-Byrne, P. (2011). Triple jeopardy: Impact of partner violence perpetration, mental health and substance use on perceived unmet need for mental health 
care among men. Social Psychiatry and Psychiatric Epidemiology, 46, 843-852. doi:10.1007/s00127-010-0258-3

Labrum, T., \& Solomon, P. L. (2015). Rates of victimization of violence committed by relatives with psychiatric disorders. Journal of Interpersonal Violence. Advance online publication. doi:10.1177/0886260515596335

Levenson, J. S., \& Socia, K. M. (2015). Adverse childhood experiences and arrest patterns in a sample of sexual offenders. Journal of Interpersonal Violence. Advance online publication. doi:10.1177/0886260515570751

Mager, K. L., Bresin, K., \& Verona, E. (2014). Gender, psychopathy factors, and intimate partner violence. Personality Disorders: Theory, Research, and Treatment, 5, 257-267. doi: $10.1037 /$ per0000072

Mair, C., Cunradi, C. B., \& Todd, M. (2012). Adverse childhood experiences and intimate partner violence: Testing psychosocial mediational pathways among couples. Annals of Epidemiology, 22, 832-839. doi:10.1016/j.annepidem.2012.09.008

Messina, N. P., Calhoun, S., \& Braithwaite, J. (2014) Trauma-informed treatment decreases PTSD among women offenders. Drug and Alcohol Dependence, 140, e147. doi:10.1016/j.drugalcdep.2014.02.416

Mersky, J. P., Topitzes, J., \& Reynolds, A. J. (2013). Impacts of adverse childhood experiences on health, mental health, and substance use in early adulthood: A cohort study of an urban, minority sample in the U.S. Child Abuse \& Neglect, 37, 917-925.

Doi:10.1016/j.chiabu.2013.07.011 
Milaniak, I., \& Widom, C. S. (2015). Does child abuse and neglect increase risk for perpetration of violence inside and outside the home? Psychology of Violence, 5, 246-255. doi:10.1037/a0037956

Millett, L. S., Kohl, P. L., Jonson-Reid, M., Drake, B., \& Petra, M. (2013). Child maltreatment victimization and subsequent perpetration of young adult intimate partner violence: An exploration of mediating factors. Child Maltreatment, 18, 71-84. doi: $10.1177 / 1077559513484821$

Piquero, A. R., Brame, R., Fagan, J., \& Moffitt, R. E. (2006). Assessing the offending activity of criminal domestic violence suspects: Offense specialization, escalation, and de-escalation evidence from the Spouse Assault Replication Program. Public Health Reports, 121, 409418.

Prins, S. J. (2014). Prevalence of mental illnesses in U.S. state prisons: A systematic review. Psychiatric Services, 65, 862-872. doi:10.1176/appi.ps.201300166

Radatz, D. L., \& Wright, E. M. (2015). Does polyvictimization affect incarcerated and nonincarcerated adult women differently? An exploration into internalizing problems. Journal of Family Violence. Advance online publication. doi:10.1177/0886260515588921

Reavis, J. A., Looman, J., Franco, K. A., \& Rojas, B. (2013). Adverse childhood experiences and adult criminality: How long must we live before we possess our own lives? The Permanente Journal, 17, 44- 48. doi:10.7812/TPP/12-072

Rice, M.E., \& Harris, G.T. (2013). Psychopathy and violent recidivism. In K.A. Kiehl \& W. Sinnott-Armstrong (Eds.) Handbook on psychopathy and law (pp. 231-249). New York: Oxford University Press. 
Roters, J., Marshall, L. E., Keating, M., \& Ball, L. (2015, June). Adverse childhood experiences. Paper presented at the Waypoint Research Institute 3rd Annual Conference, Toronto, ON.

Salekin, R. T., Rogers, R., and Sewell, K. W. (1996). A review and meta-analysis of the Psychopathy Checklist and Psychopathy Checklist-Revised: Predictive Validity of dangerousness. Clinical Psychology: Science and Practice, 3, 203-215. doi:

10.1111/j.1468-2850.1996.tb00071.x

Semiatin, J. N., Torres, S., LaMotte, A. D., Portnoy, G. A., \& Murphy, C. M. (2016). Trauma exposure, PTSD symptoms, and presenting clinical problems among male perpetrators of intimate partner violence. Psychology of Violence. Advance online publication. doi: 10.1037/vio0000041

Serin, R. C. (1996). Violent recidivism in criminal psychopaths. Law and Human Behaviour, 20, 207-217.

Smith-Marek, E. N., Cafferky, B., Dharnidharka, P. ... Mendez, M. (2015). Effects of childhood experiences of family violence on adult partner violence: A meta-analytic review. Journal of Family Theory \& Review, 7, 498-519. doi:10.1111/jftr.12113

Sorensen, J. R., Vigen, M. P., Woods, S. O., \& Williams, B. D. (2015). Assaults on inmates and staff by perpetrators of intimate partner homicide: An examination of competing hypotheses. Journal of Interpersonal Violence, 30, 3367-3388.

doi:10.1177/0886260514563831

Sullivan, E. A., Abramowitz, C. S., Lopez M., \& Kosson, D. S. (2006). Reliability and construct validity of the Psychopathy Checklist_-Revised for Latino, European American, and African American male inmates. Psychological Assessment, 18, 382-392. doi:

0.1037/1040-3590.18.4.382 
Swogger, M. T., Walsh, Z., \& Kosson, D. S. (2007). Domestic violence and psychopathic traits: Distinguishing the antisocial batterer from other antisocial offenders. Aggressive Behavior, 33, 253-260.

Swogger, M. T., Walsh, Z., Kosson, D. S., Cashman-Brown, S., \& Caine, E. D. (2012). Selfreported childhood physical abuse and perpetration of intimate partner violence. Criminal Justice and Behavior, 39, 910-922.

Teicher, M. H., \& Samson, J. A. (2016). Enduring neurobiological effects of childhood abuse and neglect. Journal of Child Psychology and Psychiatry, 57, 241 - 266. doi:10.1111/jcpp.12507

Theobald, D., Farrington, D. P., Coid, J. W., \& Piquero, A. R. (2015). Are male perpetrators of intimate partner violence different from convicted violent offenders? Examination of psychopathic traits and life success in males from a community survey. Journal of interpersonal Violence. Advance online publication. doi:10.1177/0886260515569061

Walsh, Z., Swogger, M. T., O'Connor, B. P., Schonbrun, Y. C., Shea, M. T., \& Stuart, G. L. (2010). Subtypes of partner violence perpetrators among male and female psychiatric patients. Journal of Abnormal Psychology, 119, 563-574. doi:1037/a0019858

Watt, M. \& Scrandis, D. (2013). Traumatic childhood exposures in the lives of male perpetrators of female intimate partner violence. Journal of interpersonal Violence, 28, 2813-2830. doi: $0.1177 / 0886260513488694$

Whitfield, C., Anda, R., Dube, S., \& Felitti, V. (2003). Violent childhood experiences and the risk of intimate partner violence in adults: Assessment in a large health maintenance organization. Journal of Interpersonal Violence, 18, 166-185. doi:10.1177/0886260502238733 
Table 1

Measures of Adverse Childhood Experiences (Percentage and 95\% Confidence Interval) and Criminal Propensity (Mean, Standard Deviation and 95\% Confidence Interval) as a Function of Offender Group and Correlation with ACE Score

\section{Offender Group $\quad$ Test Statistic}

\begin{tabular}{|c|c|c|c|c|c|c|}
\hline Measure & $\begin{array}{l}\text { IPV } \\
N=99\end{array}$ & $\begin{array}{l}\text { Non-IPV } \\
\mathrm{N}=233\end{array}$ & $\begin{array}{l}\text { Nonviolent } \\
\mathrm{N}=103\end{array}$ & $\chi^{2}$ or $F$ & $p$ & $r A C E$ \\
\hline \multicolumn{7}{|l|}{ ACE Experience } \\
\hline Verbal abuse & $\begin{array}{c}20 \\
{[12-28]}\end{array}$ & $\begin{array}{c}15 \\
{[10-19]}\end{array}$ & $\begin{array}{c}7 \\
{[2-12]}\end{array}$ & 7.66 & .022 & .575 \\
\hline Physical abuse & $\begin{array}{c}22 \\
{[14-31]}\end{array}$ & $\begin{array}{c}20 \\
{[15-25]}\end{array}$ & $\begin{array}{c}10 \\
{[4-16]}\end{array}$ & 6.68 & .035 & .683 \\
\hline Sexual abuse & $\begin{array}{c}9 \\
{[3-15]}\end{array}$ & $\begin{array}{c}8 \\
{[4-11]}\end{array}$ & $\begin{array}{c}3 \\
{[0-6]}\end{array}$ & 3.54 & .171 & .523 \\
\hline Neglect & $\begin{array}{c}5 \\
{[0-9]}\end{array}$ & $\begin{array}{c}6 \\
{[3-10]}\end{array}$ & $\begin{array}{c}3 \\
{[0-6]}\end{array}$ & 1.79 & .409 & .451 \\
\hline Separation from father & $\begin{array}{c}44 \\
{[34-54]}\end{array}$ & $\begin{array}{c}55 \\
{[49-61]}\end{array}$ & $\begin{array}{c}48 \\
{[38-57]}\end{array}$ & 3.62 & .163 & .548 \\
\hline Parental IPV witnessed & $\begin{array}{c}13 \\
{[6-20]}\end{array}$ & $\begin{array}{c}8 \\
{[4-11]}\end{array}$ & $\begin{array}{c}6 \\
{[1-10]}\end{array}$ & 3.86 & .146 & .542 \\
\hline $\begin{array}{l}\text { Parental substance } \\
\text { abuse }\end{array}$ & $\begin{array}{c}42 \\
{[33-52]}\end{array}$ & $\begin{array}{c}28 \\
{[23-34]}\end{array}$ & $\begin{array}{c}30 \\
{[21-39]}\end{array}$ & 6.57 & .038 & .608 \\
\hline Parental mental illness & $\begin{array}{c}27 \\
{[18-36]}\end{array}$ & $\begin{array}{c}26 \\
{[20-32]}\end{array}$ & $\begin{array}{c}25 \\
{[17-34]}\end{array}$ & 1.08 & .948 & .428 \\
\hline $\begin{array}{l}\text { Parental criminal } \\
\text { history }\end{array}$ & $\begin{array}{c}10 \\
{[4-16]}\end{array}$ & $\begin{array}{c}10 \\
{[6-14]}\end{array}$ & $\begin{array}{c}6 \\
{[1-10]}\end{array}$ & 1.84 & .399 & .527 \\
\hline Total ACE Score & $\begin{array}{c}1.94(1.66) \\
{[1.61,2.27]}\end{array}$ & $\begin{array}{c}1.76(1.82) \\
{[1.53,2.00]}\end{array}$ & $\begin{array}{c}1.37(1.61) \\
{[1.05,1.68]}\end{array}$ & 3.25 & .004 & -- \\
\hline
\end{tabular}




\begin{tabular}{|c|c|c|c|c|c|c|}
\hline $\begin{array}{l}\text { Violent criminal } \\
\text { history }\end{array}$ & $\begin{array}{c}9.54(7.61) \\
{[7.84,11.25]}\end{array}$ & $\begin{array}{l}6.55(7.29) \\
{[5.45,7.64]}\end{array}$ & $\begin{array}{l}0 \\
--\end{array}$ & 8.90 & .003 & .292 \\
\hline $\begin{array}{l}\text { Nonviolent criminal } \\
\text { history }\end{array}$ & $\begin{array}{c}2.44(1.06) \\
{[2.21,2.68]}\end{array}$ & $\begin{array}{c}1.90(1.34) \\
{[1.70,2.10]}\end{array}$ & $\begin{array}{c}1.27(1.27) \\
{[0.94,1.60]}\end{array}$ & 14.05 & $<.001$ & .231 \\
\hline Psychopathy & $\begin{array}{c}23.24(5.74) \\
{[20.63,} \\
25.86]\end{array}$ & $\begin{array}{c}18.40(7.50) \\
{[16.33} \\
20.47]\end{array}$ & $\begin{array}{c}16.42(7.65) \\
{[12.83,} \\
20.00]\end{array}$ & 5.53 & .005 & .469 \\
\hline Violent recidivism risk & $\begin{array}{l}9.01(12.61) \\
{[6.67,11.65]}\end{array}$ & $\begin{array}{l}7.71(12.10) \\
{[6.07,9.35]}\end{array}$ & $\begin{array}{l}5.42(12.90) \\
{[2.79,8.05]}\end{array}$ & 2.71 & .068 & .532 \\
\hline Institutional assaults & $\begin{array}{c}0.09(0.47) \\
{[-0.05,0.23]}\end{array}$ & $\begin{array}{c}0.94(3.55) \\
{[0.32,1.55]}\end{array}$ & $\begin{array}{c}0.20(0.67) \\
{[0.03,0.37]}\end{array}$ & 2.26 & .106 & .026 \\
\hline
\end{tabular}

Note: Criminal propensity measures represent scaled scores (Cormier Lang Criminal History, Violence Risk Appraisal Guide, and Psychopathy Checklist-Revised; see text for details) except Institutional Assaults which is a count of assaults committed within up to one year of admission. Test statistics shown are: chi-squared test of association with offender groups for dichotomous ACE items, $F$-test of two-tailed between-group differences for continuous criminal propensity measures (controling for relationship history), and correlation of each item with ACE score in the total sample. All correlation coefficients $p<.001$ except Institutional Assaults, $p=.693$. 